\title{
Calidad de vida en personas diagnosticadas con trastorno de personalidad límite: Papel mediador de la satisfacción vital
}

\author{
Beatriz Thadani, Ana M. Pérez-García y José Bermúdez \\ Universidad Nacional de Educación a Distancia, Madrid, España
}

Quality of life in patients with borderline personality disorder: The mediating role of life satisfaction

\begin{abstract}
Borderline personality disorder (BPD) is a mental illness characterized by a pattern of instability in relationships, moods and behavior. Using two groups of women (clinical or diagnosed with $\mathrm{BPD}, N=138$; and control, with no physical or mental illness, $N=124$ ) this study analyzed the differences between pathological personality traits, measured by the Personality Inventory for DSM-5 and different domains of quality of life ( $S F-36$ and the WHODAS 2.0) as well as the mediating role of life satisfaction in personality traits and quality of life. Differences were found between the groups in pathological personality and quality of life. Moreover, many SF-36 dimensions were partially mediated by life satisfaction in both groups. Therefore, new treatments for BPD may include developing life satisfaction, palliating the effects of this disorder on quality of life, reducing its impact on day-to-day tasks.
\end{abstract}

Keywords: Borderline personality disorder; personality traits; life satisfaction; quality of life; disability.

Resumen: El trastorno de personalidad límite (TPL) se caracteriza por inestabilidad en las relaciones, el humor y la conducta. Se analizaron en dos grupos de mujeres (clínico o con TPL, $N=138$; y control, sin enfermedad física o mental, $N=124$ ) las diferencias en rasgos patológicos de personalidad (evaluados con el Personality Inventory for DSM-5) y diferentes dominios de calidad de vida ( $S F-36$ y WHODAS 2.0), así como el papel mediador de la satisfacción vital en las relaciones entre personalidad y calidad de vida. Se encontraron diferencias entre los grupos en personalidad patológica y calidad de vida. Además, varias dimensiones de calidad de vida del SF-36 estaban mediadas parcialmente por la satisfacción vital en ambos grupos. Por tanto, los tratamientos del TPL podrían incluir el desarrollo de satisfacción vital para paliar sus efectos en la calidad de vida de los que lo padecen, reduciendo su impacto en las tareas del día a día.

Palabras clave: Trastorno de personalidad límite; rasgos de personalidad; satisfacción vital; calidad de vida; discapacidad.

\section{Introducción}

Los trastornos de personalidad presentan un patrón inflexible, persistente y estable a lo largo del tiempo, de experiencias internas y comportamientos, que suponen un malestar subjetivo o un deterioro de funcionamiento para las personas que los padecen, y se presentan gene-

Recibido: 23 de enero de 2018; aceptado: 04 de junio de 2018 Correspondencia: Ana M. Pérez-García, Facultad de Psicología, Universidad Nacional de Educación a Distancia, C/ Juan del Rosal, 10, Ciudad Universitaria, 28040 Madrid, España.

Correo-e: aperez@psi.uned.es ralmente en la adolescencia o al inicio de la edad adulta. Específicamente, el trastorno de personalidad límite (TPL) o personalidad borderline es un trastorno del que ha quedado patente la necesidad de incrementar las investigaciones al respecto, sobre todo de aspectos como la comorbilidad, el diagnóstico diferencial o la validación de instrumentos de medida (Fernández-Guerrero y Palacios-Vicario, 2017). Esto se vuelve más urgente al tener en cuenta que se trata de un trastorno psiquiátrico grave, en el que alrededor del $75 \%$ de los pacientes atentan contra sus vidas, y el 10\% logra culminar el suicidio (Black, Blum, Pfohl y Hale, 2004). Su piedra angular puede considerarse su patrón recurrente de inestabilidad 
en las relaciones personales, la marcada impulsividad, y las distorsiones de la autoimagen que se presentan en varios contextos de sus vidas.

Entre las características más destacables del TPL se encuentra también una severa discapacidad funcional, uso extenso de tratamientos y un empeoramiento del curso de otros trastornos depresivos que puedan también estar presentes (Leichsenring, Leibing, Kruse, New, y Leweke, 2011). Se trata también de un trastorno muy presente en los servicios de salud mental; por ejemplo, en Estados Unidos, aunque representa únicamente el 4\% en la comunidad, el TPL está presente en un $20 \%$ en las poblaciones clínicas (Kernberg y Michels, 2009). En España, su prevalencia en la población general se sitúa entre un 1-2\%, mientras que en los pacientes ambulatorios y en los hospitalizados se sitúa entre el $11-20 \%$ y el 18 $32 \%$ respectivamente. Según los datos extraídos de poblaciones españolas el TPL es tres veces más frecuente en mujeres, con una mayor prevalencia cuando se asocian un entorno urbano y un bajo nivel socioeconómico (García, Martín y Otín, 2010).

En la propuesta de modelo alternativo para la evaluación de trastornos de la personalidad presentado en el DSM-V (Personality Inventory for DSM-5), este trastorno fue uno de los seis que se incluyó (American Psychiatric Association [APA], 2013) debido en gran medida a la variedad en su manera de manifestarse, al reto que supone su tratamiento, y al gran deterioro que parece producir en los pacientes. Este modelo alternativo será el que se usará para evaluar el TPL en este estudio, ya que nos permitirá conocer y comparar con la población general las puntuaciones en personalidad patológica en las dimensiones de afectividad negativa, desapego, antagonismo, desinhibición y psicoticismo. Como otro motivo para su elección cabe destacar que el instrumento propuesto para medirlo, que cuenta con un componente dimensional, puede contribuir a desenmarañar la complejidad diagnóstica del trastorno límite al contemplar las posibles diferencias en su expresión (Smits et al., 2017).

Los rasgos de personalidad se relacionan con el estado físico del sujeto y con la percepción que se tiene del mismo. En población general, se ha encontrado que el neuroticismo se asocia negativamente con el bienestar subjetivo, mientras que la extraversión lo hace positivamente con niveles más altos de salud física y mental (Roberts, Kuncel, Shiner, Caspi, y Goldberg, 2007).

Si bien la asociación entre TPL y salud física ha sido pocas veces objetivo central de investigación sistemática, ya se dispone, no obstante, de evidencia que indica que estos pacientes presentan una historia extensa de condiciones médicas como dolor de espalda, fatiga crónica o consumo de tabaco (Frankenburg y Zanarini,
2004), así como mayor posibilidad de padecer otras enfermedades como hipertensión o artritis (El-Gabalawy, Katz y Sareen, 2010; Lee et al., 2010). Asimismo, el dolor crónico está presente en el $30 \%$ de los pacientes internos con TPL, con una representación significativamente más alta que en población general (Sansone y Sansone, 2012).

De igual modo, la patología de personalidad también afectaría a la salud percibida, pudiendo llegar a alterar las manifestaciones sintomáticas de la salud física. En el estudio de Powers y Oltmanns (2013) la personalidad borderline, al igual que la antisocial y la esquizoide, mostraba una asociación negativa con las percepciones sobre la propia salud, lo que lleva a concluir que el TPL puede actuar en detrimento de la concepción subjetiva de la salud actuando de manera indirecta sobre la salud objetiva.

La calidad de vida está muy relacionada tanto con la salud mental como con la salud física. Se considera por la Organización Mundial de la Salud (OMS) como un concepto amplio que engloba el estado físico y mental de la persona, las relaciones con los otros y con el entorno. En su artículo de revisión Ishak et al. (2013) llegaron a la conclusión de que la calidad de vida se ve seriamente afectada por la presencia del TPL pero que aquélla puede mejorar con el tratamiento.

El impacto psicológico de padecer una patología de esta complejidad y sus repercusiones a nivel físico, mental y a la postre, sobre su calidad de vida pueden traer consigo que el individuo presente una reducción de su habilidad o una discapacidad para llevar a cabo cierto tipo de tareas. Los pacientes con TPL y ansiedad comórbida presentan significativamente peor calidad de vida en los ámbitos mental, social y laboral en comparación con controles sanos (Grambal et al., 2016). Sin embargo, se necesitan más estudios al respecto que permitan llegar a un consenso sobre qué medidas utilizar para evaluar la calidad de vida en el caso de esta patología. Asimismo, se trata de un trastorno donde priman las decisiones poco ventajosas para el individuo y una tendencia a escoger opciones desadaptativas que conducen a emociones negativas (Hallguist, Hall, Schreiber, y Dombrovski, 2017). Este tipo de malas decisiones suelen reflejarse en su manera de relacionarse con los demás, en su rechazo de conductas promotoras de la salud y en verse expuestos a situaciones de riesgo que deterioran su estado físico y su calidad de vida, afectando a su manera de gestionar las relaciones, el empleo, los estudios o cualquier tipo de organización que pretendan establecer en sus vidas, lo que alteraría negativamente su funcionalidad.

En nuestro estudio para conocer este impacto, la calidad de vida será evaluada a través del World Health Organization Disability Assessment Schedule 2.0 (WHODAS 2.0) 
que entiende la calidad de vida como el grado de problemas de funcionalidad en distintas áreas, y mediante el SF-36 que evalúa la calidad de vida relacionada con la salud.

Hay que tener en cuenta que la relación entre un patrón característico de personalidad e indicadores de salud y calidad de vida seguramente no presente una relación directa. Existen factores mediadores que pueden ser clave para regular las relaciones entre una patología como el TPL, donde las cogniciones juegan un gran papel, y problemas concomitantes. Encontrar estos mediadores y trabajar en ellos podría prevenir un desarrollo negativo de la patología. Un ejemplo es el significado de la vida, que se ha encontrado que media de manera parcial el efecto de la depresión y la desesperanza en pacientes con TPL (Marco, García-Alandete, Pérez, y Botella, 2014). Las visiones subjetivas sobre la propia vida, variables importantes en el estudio del bienestar del individuo, pueden modular de manera significativa la gravedad de una condición como el trastorno de personalidad límite.

La satisfacción con la vida es un componente del bienestar subjetivo (Diener, Lucas, y Oishi, 2002). Dicho constructo viene a expresar la evaluación de la satisfacción en términos globales, de la vida en su conjunto, incluyendo los aspectos tangibles, comparaciones a partir de un criterio de elección, cómo se sopesan los aspectos positivos y negativos y los juicios cognitivos. Una alta satisfacción con la vida refleja una evaluación, o valoración subjetiva positiva de la propia vida (Ramírez y Maturana, 2012).

La satisfacción con la vida se asocia con la salud a través de su capacidad para potenciar la realización de conductas promotoras de esta. En su investigación, Grant, Wardle y Steptoe (2009) encontraron que la satisfacción con la vida se asociaba positiva y significativamente con conductas como realizar ejercicio físico, usar protector solar, no fumar y comer de manera saludable; adicionalmente se encontró que la relación entre estas conductas y la satisfacción vital no se veía afectada por las creencias de los posibles efectos beneficiosos para la salud de estas conductas.

Este componente del bienestar subjetivo también se asocia negativamente con enfermedades crónicas, conductas nocivas para la salud, una baja salud general y la discapacidad (Strine, Balluz, Chapman, y Mokdad, 2008), y positivamente con mejor estado de salud informado, resultado que se repite en diferentes países (Kööts-Ausmees y Realo, 2015). Estos datos apoyan el interés de descubrir el papel que juega la satisfacción en la calidad de vida de los pacientes con TPL.

Desde este contexto teórico, el presente estudio busca dar respuesta a dos objetivos principales. Por una parte, analizar si se confirman las diferencias entre el grupo de pacientes con TPL y un grupo control sin enfermedad mental en los diferentes dominios de la calidad de vida analizados; y por otra, estudiar si la satisfacción vital juega un papel mediador en la relación entre los rasgos de personalidad patológicos medidos en esta investigación y dichos dominios de calidad de vida.

\section{Método}

\section{Participantes}

La muestra inicial estaba formada por 294 participantes, 161 del grupo TPL y 133 del grupo control. Se eliminaron 34 casos por no cumplimentar todas las pruebas o dejar muchos ítems sin contestar, así como 8 participantes varones, para dejar la muestra sólo formada por mujeres ante el escaso número de representación masculina en el grupo clínico. En el grupo control, teniendo en cuenta esta circunstancia, se reclutaron desde el principio sólo mujeres.

La muestra final contó, entonces, con 262 mujeres (138 en el grupo clínico y 124 en el grupo control) con una edad media de 30.98 años $(D T=8.12)$ y un rango entre 18 y 55 años. El $52.7 \%$ de las participantes estaba casada o tenía pareja. En cuanto a la actividad que desarrollaban, el $27.9 \%$ eran estudiantes, el $39.7 \%$ estaban empleadas, y el $32.4 \%$ desempleadas.

Los criterios de inclusión para ambos grupos fueron tener una edad comprendida entre los 18 y los 55 años, lo que permitió controlar que cualquier deterioro detectado no se debiera a una avanzada edad, y tener una buena comprensión de la lengua española, para cumplimentar apropiadamente las pruebas. En cuanto a los criterios específicos, las participantes del grupo control no debían presentar ninguna enfermedad física o mental, estar en terapia psicológica o tomar medicación psiquiátrica. También debían informar de una salud física y mental de al menos 3 en una escala de 5 puntos. En cuanto al grupo TPL, el único requisito específico era estar diagnosticado por un profesional de la salud mental (psicólogo o psiquiatra) con trastorno de personalidad límite. El 9.5\% llevaba diagnosticado menos de 6 meses; el 32.5\% entre 6 meses y 2 años, el $25.2 \%$ entre 2 y 5 años, y el $32.5 \%$ restante, más de 5 años. El 57.8\% había sido diagnosticado en centros de la sanidad pública y el $42.2 \%$ en centros de la sanidad privada.

\section{Instrumentos}

Para medir la personalidad, se utilizó el Personality Inventory for DSM-5 - Brief Form (PID-5-BF; American Psychiatric Association [APA], 2013); se trata de un ins- 
trumento de autoinforme que tiene en cuenta las dificultades particulares del individuo en el funcionamiento de la personalidad y el patrón de los rasgos específicos que evalúa. Esta versión consta de un total de 25 ítems, distribuidos en 5 dimensiones con 5 ítems cada una: Afectividad negativa («Me emociono fácilmente incluso por el más mínimo motivo»), Desapego («No me gusta acercarme mucho a la gente»), Antagonismo («No me importa demasiado herir los sentimientos de otros»), Desinhibición («La gente diría que soy imprudente») y Psicoticismo ( $«$ Con frecuencia tengo pensamientos que tienen sentido para mí pero que otros dicen que son extraños»). Los ítems se evalúan según el grado de acuerdo con cada enunciado en una escala de 4 puntos (siendo 0 «bastante falso» y 3 «bastante verdadero»). Permite obtener una puntuación total en personalidad patológica y específica en cada dimensión. La versión española de la escala ampliada mostró una consistencia interna media de .86 en muestra clínica y .79 en muestra control, así como capacidad para distinguir entre ambos tipos de muestras (Gutiérrez et al., 2015). En la presente muestra se obtuvieron índices de consistencia interna (alfa de Cronbach) entre .78 para Antagonismo y .86 para Psicoticismo, así como .95 para la escala total.

Para evaluar, dentro de la calidad de vida, la funcionalidad y discapacidad se utilizó el World Health Organization Disability Assessment Schedule 2.0. (WHODAS 2.0; World Health Organization [WHO], 2001; versión en español de Vázquez-Barquero et al., 2000), escala autoadministrada de 36 ítems, que mide la salud, la discapacidad y las limitaciones a partir de las experiencias de los últimos 30 días en las siguientes categorías: Comunicación, Movilidad, Cuidado personal, Relaciones con otras personas, Actividades de la vida diaria y Participación en sociedad. Se evalúa la dificultad encontrada para realizar lo que se postula en cada pregunta, en una escala de 5 puntos (siendo 0 «ninguna o nada» y 4 «extrema o no puede hacerlo»). Se puede obtener la puntuación total en la escala, así como en cada una de las categorías evaluadas. Se considera un instrumento robusto para conocer el peso de los trastornos físicos y mentales en la vida diaria de las personas. Se ha informado una consistencia interna media de .86 (Üstün, Kostanjsek, Chatterji y Rehm, 2010) y de .96 (Guilera et al., 2015). En la presente muestra se consideró únicamente la puntuación total en la escala, con una consistencia interna de .97, como medida de Discapacidad.

Para los demás aspectos de la calidad de vida, relacionados con la salud física y psicológica se utilizó la Short Form Health Survey (SF-36; Ware, Snow, Kosinski, y Gandek, 1993; versión en español de Alonso, Prieto y Anto, 1995), uno de los cuestionarios autoadministra- dos más utilizados para medir la calidad de vida relacionada con la salud. Sus 36 ítems, que se responden en diferentes escalas cuyas puntuaciones luego se transforman, están agrupados en 8 secciones que miden Funcionamiento físico (grado en que la salud física limita las actividades físicas), Funcionamiento del rol físico (grado en que la salud física interfiere en las actividades diarias), Funcionamiento del rol emocional (grado en que los problemas emocionales interfieren en las actividades diarias), Vitalidad (sentimiento de energía frente al cansancio o agotamiento), Salud mental (incluyendo problemas de depresión, ansiedad, control de la conducta y control emocional), Funcionamiento del rol social (grado en que los problemas de salud física o emocional interfieren en la vida social habitual), Salud general (valoración personal de la salud), y Dolor corporal. Todas las escalas se transforman de manera que, a mayor puntuación, mayor calidad de vida relacionada con la salud, es decir, menos limitaciones, menos dolor, mejor funcionamiento en las diferentes esferas consideradas, etc. La revisión de Vilagut et al. (2005) encontró para la versión española datos de consistencia interna de las diferentes subescalas superiores a .70. En la presente muestra se obtuvieron valores entre .77 para Funcionamiento del rol social y .94 para el Funcionamiento del rol emocional, con un promedio de .88 .

Finalmente, para evaluar la satisfacción vital se utilizó la Satisfaction with Life Scale (SWLS; Diener, Emmons, Larsen, y Griffin, 1985; versión en español de Atienza, Pons, Balaguer y García-Merita, 2000), escala autoadministrada de 5 ítems evaluados en escalas de 7 puntos en función del acuerdo con cada enunciado $(1$ = ningún acuerdo, $7=$ total acuerdo). Las altas puntuaciones reflejan satisfacción general con la vida, sin considerar de forma específica ningún contexto en particular. Se han informado datos de consistencia interna entre .79 y .89, para la versión original (Pavot y Diener, 1993) y de .84, para la versión española (Atienza et al., 2000). En la presente muestra se obtuvo una consistencia interna de .92 .

\section{Procedimiento}

En este estudio, de carácter transversal, las participantes completaron los cuestionarios en una única ocasión, a través de dos plataformas online, una para cada grupo. Al acceder a sus respectivas páginas las participantes rellenaron un breve cuestionario de inscripción en el que se les planteaban las cuestiones de carácter sociodemográfico y se explicaban todos los términos del estudio. Para completar este registro las participantes dieron su consentimiento para ser incluidas en esta investigación. Una vez completada esta inscripción se les 
daba acceso a las pruebas que cumplimentaron y enviaron a través de la página web donde posteriormente se almacenaron en la base de datos.

La muestra de afectadas por TPL se obtuvo con la ayuda de tres asociaciones especializadas en trastornos de la personalidad que accedieron a invitar a sus usuarias a participar en el estudio, así como a publicar en sus grupos de redes sociales el enlace a la web de recogida de datos. Las asociaciones que cooperaron fueron: La Fundación Regional Murciana de Ayuda e Investigación del Trastorno Límite de la Personalidad (ARMAI-TPL), Trastornos de Personalidad Galicia (TP-Galicia) y La Asociación Valenciana de Trastorno Límite de la Personalidad (ASVA-TP). En cuanto al grupo de control se formó con mujeres que estudiaban en la UNED, ya que las características de esta universidad permiten contar con un rango amplio de edades, además de desempeñar muchas de ellas también una actividad laboral. En todos los casos se contó con informe favorable de las asociaciones y de la universidad sobre el proyecto investigador y la protección de datos de las participantes.

\section{Análisis estadístico}

Se utilizó el programa SPSS 24 para realizar los análisis estadísticos. Las diferencias entre los grupos clínico y control se analizaron con la prueba de $\chi^{2}$ para las variables sociodemográficas, y con la $t$ de Student para las variables psicológicas. El posible papel mediador de la satisfacción vital, en las relaciones de la personalidad patológica con los diferentes criterios de calidad de vida medidos, se contrastó mediante regresiones lineales múltiples jerárquicas. En aquellos casos en los que la satisfacción vital resultó un predictor significativo, se analizó si había efecto de mediación utilizando el test de Sobel.

\section{Resultados}

Los datos sociodemográficos de las participantes en el estudio (ver Tabla 1) indican que no hubo diferencias en edad entre los dos grupos $(p=.25)$, aunque sí había más mujeres sin pareja $\left(\chi^{2}=9.89, p=.002, V\right.$ de Cramer $=.19)$ y desempleadas $\left(\chi^{2}=20.57, p<.001, V\right.$ de Cramer $=.28)$ en el grupo clínico.

La tabla 2 presenta los datos descriptivos de las variables estudiadas para los dos grupos analizados, así como la prueba $t$ de Student, tomando en cada caso el valor que correspondía según fuera significativa o no la prueba de Levene para la igualdad de las varianzas. En todos los contrastes, las diferencias fueron significati-
Tabla 1. Características sociodemográficas de los grupos del estudio.

\begin{tabular}{lcc}
\cline { 2 - 3 } & $\begin{array}{c}\text { Grupo clínico } \\
n=138)\end{array}$ & $\begin{array}{c}\text { Grupo control } \\
n=124)\end{array}$ \\
\hline $\begin{array}{l}\text { Edad [media }(D T)] \\
\text { Pareja }[n(\%)]\end{array}$ & $30.44(8.08)$ & $31.59(8.15)$ \\
No & $78(56.5)$ & $48(37.1)$ \\
Sí & $60(43.5)$ & $78(62.9)$ \\
Actividad $[n(\%)]$ & & \\
Estudiantes & $39(28.3)$ & $34(27.4)$ \\
Empleadas & $39(28.3)$ & $65(52.4)$ \\
No empleadas & $60(43.5)$ & $25(20.2)$ \\
\hline
\end{tabular}

vas $(p<.001)$ y con tamaños del efecto muy grandes, mostrando el grupo clínico, en comparación con el control, puntuaciones más altas en los rasgos de personalidad patológica y en discapacidad, y más bajas en satisfacción vital y en los dominios de calidad de vida medidos con el SF-36.

A continuación, y dadas las importantes diferencias encontradas entre ambos grupos, se analizó, para cada uno de ellos por separado, el efecto mediacional de la satisfacción vital en las relaciones de la personalidad patológica (puntuación total en la escala PID-5-BF) con los criterios de calidad de vida recogidos, como problemas de discapacidad o de funcionalidad (puntuación total en la prueba WHODAS) y los diferentes dominios de la prueba SF-36. En las regresiones lineales múltiples jerárquicas se introdujo en el Paso 1 la personalidad, considerada como la variable independiente (VI) en el análisis mediacional, y se añadió en el paso 2 la satisfacción vital, considerada como variable mediadora (VM). No se encontraron efectos de colinealidad en ninguno de los análisis realizados. En la tabla 3 pueden consultarse los datos de los modelos finales (Paso 2), así como observar el cambio de la beta estandarizada de la VI (personalidad) entre el paso 1 y el paso 2 en el que se añade el efecto del mediador (satisfacción vital), junto con el test de Sobel, en los casos en que la VM resultó significativa.

Las relaciones entre personalidad y el dominio de discapacidad resultaron directas en el grupo clínico, mientras que en el grupo control estaban parcialmente mediadas por la satisfacción vital $(p<.001)$. En cuanto a las relaciones entre personalidad y los dominios medidos en el SF-36, estaban parcialmente mediadas por la satisfacción vital, en ambos grupos, la vitalidad $(p<.01)$ y la salud mental $(p<.001)$, y en el grupo control, la salud general $(p<.01)$. En cuanto al funcionamiento del 
Tabla 2. Descriptivos (media y desviación típica) de los grupos clínico $(n=138)$ y control $(n=124)$, prueba de contraste entre grupos ( $t$ de Student) y tamaño del efecto ( $d$ de Cohen)

\begin{tabular}{lcccc}
\hline & Grupo clínico & Grupo control & $t$ & $d$ \\
\hline PID-5 Afectividad negativa & $2.39(.51)$ & $1.14(.58)$ & $18.31^{* * *}$ & 2.29 \\
PID-5 Desapego & $1.68(.64)$ & $0.61(.52)$ & $14.72^{* * *}$ & 1.84 \\
PID-5 Antagonismo & $1.50(.67)$ & $0.48(.40)$ & $15.12^{* * *}$ & 1.85 \\
PID-5 Desinhibición & $2.17(.56)$ & $0.72(.58)$ & $20.49^{* * *}$ & 2.46 \\
PID-5 Psicoticismo & $1.91(.65)$ & $0.62(.53)$ & $17.83^{* * *}$ & 2.19 \\
PID-5 TOTAL & $1.93(.41)$ & $0.72(.37)$ & $24.99^{* * *}$ & 3.12 \\
SATISFACCIÓN TOTAL & $2.37(1.15)$ & $4.60(1.17)$ & $-15.49^{* * *}$ & -1.92 \\
WHODAS DISCAPACIDAD TOTAL & $2.97(.64)$ & $1.40(.41)$ & $23.84^{* * *}$ & 2.92 \\
SF Funcionamiento físico & $78.80(22.14)$ & $95.44(8.67)$ & $-8.16^{* * *}$ & -1.00 \\
SF Funcionamiento rol físico & $50.91(45.45)$ & $85.48(30.09)$ & $-7.33^{* * *}$ & -0.90 \\
SF Funcionamiento rol emocional & $7.97(24.68)$ & $69.62(41.43)$ & $-14.43^{* * *}$ & -1.81 \\
SF Vitalidad & $28.22(18.42)$ & $58.51(16.54)$ & $-13.94^{* * *}$ & -1.73 \\
SF Salud mental & $33.30(16.11)$ & $67.48(16.49)$ & $-16.96^{* * *}$ & -2.10 \\
SF Funcionamiento rol social & $55.47(26.84)$ & $78.37(18.37)$ & $-8.12^{* * *}$ & -1.00 \\
SF Salud general & $38.12(22.19)$ & $68.67(18.06)$ & $-12.27^{* * *}$ & -1.51 \\
SF Dolor (ausencia) & $55.47(26.84)$ & $78.37(18.37)$ & $-8.12^{* * *}$ & -1.00 \\
\hline
\end{tabular}

Nota . PID-5 = Personality Inventory for DSM-5; WHODAS = WHO Disability Assessment Schedule; SF = Short Form Health Survey; $\mathrm{M}=$ Media; $\mathrm{DT}=$ Desviación típica; ${ }^{* * *} p<.001$.

rol emocional, las relaciones estaban mediadas por la satisfacción de forma parcial en el grupo control $(p<.05)$ y total en el grupo clínico $(p<.01)$ (Tabla 3$)$.

\section{Discusión}

El primer objetivo de este estudio fue analizar las diferencias entre un grupo de afectadas por TPL y un grupo control en rasgos de personalidad patológicos, calidad de vida y satisfacción vital. Los resultados mostraron, además de las esperadas puntuaciones más extremas en los cinco rasgos patológicos de personalidad medidos, menor calidad de vida en las pacientes, en todos los dominios, resultados que apuntan hacia un deterioro físico y funcional en quienes padecen este tipo de patologías.

En los trastornos de personalidad, las conductas de salud y las elecciones en el estilo de vida características suelen conducir a problemas médicos y al empeoramiento de condiciones físicas ya existentes (Douzenis, Tsopelas y Tzeferakos, 2012). Por ejemplo, el inicio del tratamiento de pacientes con trastorno por dependencia a la cocaína se dificulta notablemente si presentan baja calidad de vida y además padecen trastornos de personalidad, al verse disminuidos los recursos necesarios para afrontar todo el proceso (Martínez-González, Albein-Urios, Lozano-Rojas y Verdejo-García, 2015). Incluso se ha encontrado evidencia a favor de que cuanto mayor es el número de criterios que se cumplen para un trastorno de personalidad, más baja es la calidad de vida, presentando los afectados más discapacidades a la hora de funcionar y comportarse en diferentes ámbitos (Cramer, Torgersen,y Kringlen, 2006).

En el caso concreto del TPL, y como ocurre en el presente estudio, los pacientes presentan una significativa menor calidad de vida relacionada con la salud (IsHak et al., 2013). Por ejemplo, se han informado valores entre .48 y .52 en el EuroQol-5D (EQ-5D), mientras que en la población general el rango se encuentra entre .83 y .87 (Van Asselt, Dirksen, Arntz, Giesen-Bloo, y Severens, 2009).

En cuanto a la discapacidad funcional, los datos obtenidos confirman resultados previos acerca de la gran incapacitación de estos pacientes a la hora de desarrollar actividades laborales, en las relaciones sociales y en las actividades de ocio, incluso más que las limitaciones funcionales comúnmente asociadas a los otros trastornos de personalidad o a los trastornos depresivos (Skodol et al., 2002). Profundizando en la interacción social, Jeung y Herpertz (2014) concluyeron que estos pacientes su- 
Tabla 3. Modelo final de las regresiones lineales múltiples jerárquicas y test de Sobel de efectos mediacionales de la satisfacción vital en las relaciones de la personalidad (PID-5-BF) con los diferentes dominios de la calidad de vida medidos en los grupos clínico y control.

\begin{tabular}{|c|c|c|c|c|c|}
\hline Criterios [VD] & $\beta[\mathrm{VI}]$ Personalidad & $\beta[\mathrm{VM}]$ Satisfacción & $F$ & $R^{2}$ & Sobel (Z) \\
\hline \multicolumn{6}{|l|}{ Grupo clínico } \\
\hline Discapacidad total & $.54^{* * *}\left(.57^{* * *}\right)$ & -.07 & $32.91^{* * *}$ & .32 & \\
\hline SF Func. físico & $-.27^{* * *}\left(-.27^{* * *}\right)$ & -.01 & $5.19^{* *}$ & .06 & \\
\hline SF Func. rol físico & $-.02(-.06)$ & -.09 & .68 & .01 & \\
\hline SF Func. rol emocional & $-.08\left(-.18^{*}\right)$ & $.26^{* *}$ & $6.50^{* *}$ & .07 & $-2.47^{* *}$ \\
\hline SF Vitalidad & $-.32^{* * *}\left(-.41^{* * *}\right)$ & $.25^{* *}$ & $19.29^{* * *}$ & .21 & $-2.57^{* *}$ \\
\hline SF Salud mental & $-.29^{* * *}\left(-.43^{* * *}\right)$ & $.37^{* * *}$ & $29.91^{* * *}$ & .30 & $-3.40^{* * * *}$ \\
\hline SF Func. rol social & $-.31^{* * *}\left(-.29^{* * *}\right)$ & -.06 & $6.42^{* *}$ & .07 & \\
\hline SF Salud general & $-.33^{* * *}\left(-.38^{* * *}\right)$ & .13 & $12.62^{* * *}$ & .15 & \\
\hline SF Dolor (ausencia) & $-.31^{* * *}\left(-.29^{* * *}\right)$ & -.06 & $6.42^{* *}$ & .07 & \\
\hline \multicolumn{6}{|l|}{ Grupo control } \\
\hline Discapacidad total & $.43^{* * *}\left(.59^{* * *}\right)$ & $-.35^{* * *}$ & $46.97^{* * *}$ & .43 & $3.49^{* * *}$ \\
\hline SF Func. físico & $-.11(-.17)$ & .13 & 2.56 & .03 & \\
\hline SF Func. rol físico & $-.06(-.10)$ & .09 & 1.01 & .00 & \\
\hline SF Func. rol emocional & $-.27^{* *}\left(-.37^{* * *}\right)$ & $.23^{*}$ & $12.90^{* * *}$ & .16 & $-2.23^{*}$ \\
\hline SF Vitalidad & $-.39^{* * *}\left(-.50^{* * *}\right)$ & $.24^{* *}$ & $25.04^{* * *}$ & .28 & $-2.53^{* *}$ \\
\hline SF Salud mental & $-.44^{* * *}\left(-.59^{* * *}\right)$ & $.33^{* * *}$ & $46.50^{* * *}$ & .43 & $-3.42^{* * *}$ \\
\hline SF Func. rol social & $-.30^{* *}\left(-.30^{* *}\right)$ & -.01 & $5.83^{* *}$ & .07 & \\
\hline SF Salud general & $-.26^{* *}\left(-.37^{* * *}\right)$ & $.26^{* *}$ & $14.30^{* * *}$ & .18 & $-2.50^{* *}$ \\
\hline SF Dolor (ausencia) & $-.30^{* *}\left(-.30^{* *}\right)$ & -.01 & $5.83^{* *}$ & .07 & \\
\hline
\end{tabular}

Nota. ${ }^{*} p<.05 ;{ }^{* *} p<.01 ;{ }^{* * *} p<.001$. El valor de $R^{2}$ es el corregido o ajustado. $\beta=$ beta estandarizada. En la columna del factor Personalidad se recoge entre paréntesis la beta estandarizada del paso 1 del análisis de regresión. Func. = funcionamiento. VI = variable independiente, $\mathrm{VM}=$ variable mediadora $\mathrm{y} \mathrm{VD}=$ variable dependiente

fren una severa disfunción sobre todo en el ámbito de las relaciones románticas.

Por lo que se refiere a la salud física, los trastornos de personalidad tienen efectos a largo plazo y de carácter significativo, como un mayor riesgo de enfermedades crónicas o una mayor mortalidad. Padecer un trastorno de personalidad es pues un predictor significativo de un funcionamiento físico mermado, fatiga, limitaciones de rol y dolor, así como de un mayor uso de los servicios médicos (Powers y Oltmanns, 2013).

En el TPL se ha encontrado que el riesgo de padecer enfermedades físicas crónicas es menor en los pacientes que están en remisión que en los que siguen padeciendo el trastorno, así como que la incapacidad para recuperarse de este trastorno deriva en la tendencia a tomar decisiones en el estilo de vida perjudiciales para la salud y en un mayor riesgo de padecer enfermedades crónicas (Frankenburg y Zanarini, 2004).
El segundo objetivo del estudio fue conocer el papel mediador de la satisfacción vital en las relaciones entre TPL y los diversos indicadores de calidad de vida evaluados. La satisfacción no medió la relación entre personalidad y discapacidad globalmente considerada en el grupo de pacientes con TPL, aunque sí lo hizo, de manera parcial, en el grupo control. Por su parte, en el grupo clínico, el peso de los rasgos estables, es decir, de la personalidad patológica, eclipsa el potencial papel de la satisfacción vital a la hora de explicar las limitaciones y los problemas en el día a día en todas las áreas. Esto puede deberse al gran impacto que tiene esta patología para mermar la capacidad del individuo para funcionar normalmente en la vida. En el grupo control, sin embargo, la personalidad no predecía significativamente el funcionamiento físico, resultado que iría en la línea de otros obtenidos también en poblaciones no clínicas, como estudiantes universitarios (Riveros y Vinaccia-Alpi, 2017). 
No obstante, en el grupo TPL, al igual que en el grupo control, se encontraron dimensiones del SF-36 mediadas por la satisfacción vital. Estos resultados son coherentes con la literatura al respecto, en estudios de población general. En apoyo a estas mediaciones, puede citarse el estudio de Strein, Chapman, Balluz, Moriarty, y Mokdad (2008) que investigó la asociación entre la satisfacción vital y la calidad de vida relacionada con la salud, y advirtió que a medida que el nivel de satisfacción se veía reducido, aumentaba la mala salud general, la discapacidad y la falta de apoyo social, viéndose incrementado también el malestar físico y mental, la escasa actividad y el dolor. Asimismo, Grant et al. (2009) hallaron que este componente del bienestar subjetivo muestra una asociación positiva con conductas de salud como la realización de ejercicio y el desarrollo de una alimentación saludable.

Puede que las divergencias encontradas entre los resultados que hemos obtenido en el caso de la discapacidad y los informados en la literatura estén relacionadas con las características de nuestra muestra, al estar formada únicamente por mujeres y tratarse de un grupo clínico, mientras que en los estudios mencionados se incluían ambos sexos y muestras de población general no clínica. De hecho, los resultados obtenidos en el grupo control sí confirman la mediación por parte de la satisfacción en discapacidad total en el WHODAS 2.0, mientras que en el grupo clínico existía una relación directa entre personalidad patológica y este constructo.

Este estudio no está exento de limitaciones. Una de ellas es que se tiene en cuenta sólo a mujeres, lo que podría hacer que se perdiera gran parte de la varianza que estaría presente en una muestra que considerara ambos sexos.

El carácter transversal de este estudio es otro de sus inconvenientes, ya que no podemos conocer cómo evolucionan en una misma muestra las relaciones entre el TPL, la discapacidad, la calidad de vida relacionada con la salud y la satisfacción vital a lo largo del tiempo, sobre todo teniendo en cuenta la importancia de la edad en este trastorno, ya que sus síntomas parecen perder agresividad con el paso de los años (Biskin, 2015). Por ello sería de gran interés continuar el estudio desde una perspectiva longitudinal que además nos permitiera establecer relaciones causales entre las variables analizadas.

En lo que respecta a las aportaciones de esta investigación, este estudio ha ahondado en un tema sobre el cual existe aún poca investigación. Escasos estudios se han centrado en el impacto del trastorno de personalidad límite sobre la calidad de vida de estos pacientes, por lo que cualquier investigación que se centre en este ámbito es útil y relevante para arrojar más luz sobre esta relación.
El conocimiento del daño que esta patología puede hacer en otras dimensiones de la vida de quienes la padecen y el nivel de discapacidad que involucra puede ser de gran ayuda a la hora de llevar a cabo un tratamiento apropiado. Por ejemplo, en este estudio se ha confirmado la gran discapacidad que presentan estas personas en diversas áreas de sus vidas, pero también en sus relaciones interpersonales. El conocimiento de esta habilidad relacional mermada puede permitir a los terapeutas otorgar un trato y un enfoque que tenga en cuenta estas limitaciones a la hora de tratar a estos pacientes, ya que actualmente, el presentar un diagnóstico de TPL en muchos casos puede interferir con la consolidación de una adecuada relación terapéutica, lo que en ocasiones deriva en prácticas clínicas poco beneficiosas para estos pacientes (Jeung y Herpertz, 2014).

Asimismo, si tenemos en cuenta los problemas físicos que padecen estas personas podremos tratarlos antes de que deriven en condiciones más graves y crónicas. De igual forma, si consideramos la alta discapacidad que muestran a la hora de llevar a cabo tareas cotidianas, podremos incluir en su tratamiento herramientas y estrategias que les permitan recuperar cierto grado de funcionalidad y por lo tanto aumentar su calidad de vida.

Por otra parte, se puede abrir el camino a una detección temprana del trastorno límite, ya que en atención primaria se puede plantear la posibilidad de que personas que presenten alta discapacidad acompañada de problemas de salud puedan padecer TPL y ser derivadas a un profesional de la salud mental en caso de que fuera necesario.

Es significativo conocer el papel que puede tener la satisfacción vital en la modulación de las relaciones que hemos estudiado. Ya que este elemento del bienestar subjetivo ha mostrado la capacidad de mediar entre el TPL y dimensiones de calidad de vida, sería de utilidad trabajarlo con los pacientes con trastorno límite en terapia, sobre todo teniendo en cuenta que los niveles de satisfacción con la vida pueden ser modificados mediante intervenciones de psicología positiva (Sin y Lyubomirsky, 2009), lo que a su vez puede aliviar la sintomatología y mejorar la calidad de vida relacionada con la salud de estos pacientes (IsHak et al., 2013).

Para seguir ahondando en la relación entre TPL, calidad de vida y nivel de incapacitación, sería necesario ampliar la muestra incluyendo ambos sexos y un grupo con otro trastorno de personalidad. Ello permitiría comparar el posible efecto diferencial de ambos trastornos sobre estas variables, además de considerar la influencia del sexo en dichas relaciones, pudiendo establecer patrones con las idiosincrasias propias de esta enfermedad. 


\section{Conflicto de intereses}

Los autores declaran no tener ningún conflicto de intereses.

\section{Referencias}

Alonso, J., Prieto, L., y Anto, J. M. (1995). La versión española del SF-36 Health Survey (Cuestionario de Salud SF-36): Un instrumento para la medida de los resultados clínicos. Medicina Clínica, 104, 771-776

Atienza, F. A., Pons, D., Balaguer, I., y García-Merita, M. (2000). Propiedades psicométricas de la Escala de Satisfacción con la Vida en adolescentes. Psicothema, 12, 314-319.

American Psychiatric Association [APA]. (2013). Diagnostic and statistical manual of mental disorders (5th ed.). Arlington, VA: APA. https://doi.org/10.1176/appi.books.9780890425596

Biskin, R. S. (2015). The lifetime course of borderline personality disorder. The Canadian Journal of Psychiatry, 60, 303-308. https://doi.org/10.1177/070674371506000702

Black, D.W., Blum, N., Pfohl, B., y Hale, N. (2004). Suicidal behavior in borderline personality disorder: Prevalence, risk factors, prediction, and prevention. Journal of Personality Disorders, 18 , 226-239. https://doi.org/10.1521/pedi.18.3.226.35445

Cramer, V., Torgersen, S., y Kringlen, E. (2006). Personality disorders and quality of life. A population study. Comprehensive Psychiatry, 47, 178-184. https://doi.org/10.1016/j.comppsych. 2005.06.002

Diener, E., Emmons, R. A., Larsen, R. J., y Griffin, S. (1985). The Satisfaction with Life Scale. Journal of Personality Assessment, 49, 71- 75. https://doi.org/10.1207/s15327752jpa4901_13

Diener, E., Lucas, R. E., y Oishi, S. (2002). Subjective well-being: The science of happiness and life satisfaction. En C. R. Snyder y S. J. Lopez (Ed.), Handbook of positive psychology (pp. 63-73). New York: Oxford University Press.

Douzenis, A., Tsopelas, C., y Tzeferakos, G. (2012). Medical comorbidity of cluster B personality disorders. Current Opinion in Psychiatry, 25, 398-404. https://doi.org/10.1097/ YCO.0b013e3283558491

El-Gabalawy, R., Katz, L.Y., y Sareen, J. (2010). Comorbidity and associated severity of borderline personality disorder and physical health conditions in a nationally representative sample. Psychosomatic Medicine, 72, 641-647. https://doi. org/10.1097/PSY.0b013e3181e10c7b

Fernandez-Guerrero, M. J. y Palacios-Vicario, B. (2017). El trastorno de personalidad límite en la producción científica publicada en revistas editadas en España. Clínica y Salud, 28, 147-153. https://doi.org/10.1016/j.clysa.2017.05.002

Frankenburg, F. R. y Zanarini, M. C. (2004). The association between borderline personality disorder and chronic medical illnesses, poor health-related lifestyle choices, and costly forms of health care utilization. Journal of Clinical Psychiatry, 12, 1660-1665. https://doi.org/10.4088/JCP.v65n1211

García, M. T., Martín, M. F., y Otín, R. (2010). Tratamiento integral del trastorno de personalidad límite. Revista de la Asociación Española de Neuropsiquiatría, 30, 263-278. https://doi.org/10.4321/S0211-57352010000200005
Guilera, G., Gómez-Benito, J., Pino, O., Rojo, E., Vieta, E., Cuesta, M.J., ...Rejas, J. (2015). Disability in bipolar 1 disorder: The 36-item World Health Organization Disability Assessment Schedule 2.0. Journal of Affective Disorders, 174, 353-360. https://doi.org/10.1016/j.jad.2014.12.028

Grambal, A., Prasko, J., Kamaradova, D., Latalova, K., Holubova, M., Sedláčková Z., y Hruby, R. (2016). Quality of life in borderline patients comorbid with anxiety spectrum disorders - a cross-sectional study. Patient Preference and Adherence, 10, 1421-1433. https://doi.org/10.2147/PPA.S108777

Grant, N., Wardle, J., y Steptoe, A. (2009). The relationship between life satisfaction and health behavior: A cross-cultural analysis of young adults. International Journal of Behavioral Medicine, 16, 259-68. https://doi.org/10.1007/s12529-0099032-x

Gutiérrez, F., Aluja, A., Peri, J. M., Calvo, N., Ferrer, M., GutiérrezZotes, J. A., ...Krueger, R. F. (2015). Psychometric properties of the Spanish PID-5 in a clinical and a community sample. Assessment, 22, 1-11.https://doi.org/10.1177/1073191115606518

Hallquist, M. N., Hall, N. T., Schreiber, A. M., y Dombrovski, A.Y. (2017). Interpersonal dysfunction in borderline personality: A decision neuroscience perspective. Current Opinion in Psychology, 21, 94-104. https://doi.org/10.1016/j. copsyc.2017.09.011

IsHak, W. W., Elbau, I., Ismail, A., Delaloye, S., Ha, K., Bolotaulo, N. I., ...Wang, C. (2013). Quality of life in borderline personality disorder. Harvard Review of Psychiatry, 21, 138150. https://doi.org/10.1097/HRP.0b013e3182937116

Jeung, H. y Herpertz, S. C. (2014). Impairments of interpersonal functioning: Empathy and intimacy in borderline personality disorder. Psychopathology, 47, 220-234. https://doi. org/10.1159/000357191

Kernberg, O. F. y Michels, R. (2009). Borderline personality disorder. American Journal of Psychiatry, 166, 505-508. https://doi.org/10.1176/appi.ajp.2009.09020263

Kööts-Ausmees, L. y Realo, A. (2015). The association between life satisfaction and self-reported health status in Europe. European Journal of Personality, 29, 647-657. https://doi. org/10.1002/per.2037

Lee, H., Bienvenu, J., Cho, S., Ramsey, C., Bandeen-Roche, K., Eaton, W. W., y Nestadt, G. (2010). Personality disorders and traits as predictors of incident cardiovascular disease: Findings from the 23-year follow-up of the Baltimore ECA Study. Psychosomatics: Journal of Consultation Liaison Psychiatry, 51,289-296. https://doi.org/10.1016/S0033-3182(10)70699-X

Leichsenring, F., Leibing, E., Kruse, J., New, S. A., y Leweke, F. (2011). Borderline personality disorder. The Lancet, 377, 7484. https://doi.org/10.1016/S0140-6736(10)61422-5

Marco, J. H., García-Alandete, J., Pérez, S., y Botella, C. (2014). El sentido de la vida como variable mediadora entre la depresión y la desesperanza en pacientes con trastorno límite de la personalidad. Behavioral Psychology, 22, 293-305. http://hdl.handle.net/10234/120322

Martínez-González, J. M., Albein-Urios, N., Lozano-Rojas, O., y Verdejo-García, A. (2015). Variables de interés clínico en el tratamiento cognitivo-conductual de la adicción a la cocaína: Especificidad de los trastornos de la personalidad. Revista de Psicopatología y Psicología Clínica, 20, 115-123. https://doi. org/10.5944/rppc.vol.20.num.2.2015.15166 
Pavot, W. y Diener. E. (1993). The affective and cognitive contest of self-reports measures of subjective well-being. Social Indicators Research, 28, 1-20. https://doi.org/10.1007/ BF01086714

Powers, A. D. y Oltmanns, T. F. (2013). Personality pathology as a risk factor for negative health perception. Journal of Personality Disorders, 27, 359-370. https://doi.org/10.1521/ pedi_2013_27_083

Ramírez, P. M. y Maturana, S. L. (2012). Factores asociados a la satisfacción vital en adultos mayores de 60 años. Revista de la Universidad Bolivariana, 11, 407-427. https://doi. org/10.4067/S0718-65682012000300020

Riveros, F. y Vinaccia-Alpi, S. (2017). Relación entre variables sociodemográficas, patogénicas y salutogénicas, con la calidad de vida en estudiantes universitarios colombianos. Revista de Psicopatología y Psicología Clínica, 22, 229-241. https://doi.org/10.5944/rppc.vol.22.num.3.2017.16330

Roberts, B. W., Kuncel, N. R., Shiner, R., Caspi, A., y Goldberg L. R. (2007). The power of personality. The comparative validity of personality traits, socioeconomic status, and cognitive ability for predicting important life outcomes. Perspectives on Psychological Science, 2, 313-345. https://doi. org/10.1111/j.1745-6916.2007.00047.x.

Sansone, R. A. y Sansone, L. A. (2012). Chronic pain syndromes and borderline personality. Innovations in Clinical Neuroscience, 9, 10-14.

Sin, N. L. y Lyubomirsky, S. (2009). Enhancing well-being and alleviating depressive symptoms with positive psychology interventions: A practice-friendly meta-analysis. Journal of Clinical Psychology, 65, 467-487. https://doi.org/10.1002/ jclp.20593

Skodol, A. E., Gunderson, J. G., McGlashan, T. H., Dyck, I. R., Stout, R. L., Bender, D. S., ... Oldham, J. M. (2002). Functional impairment in patients with schizotypal, borderline, avoidant, or obsessive-compulsive personality disorder. American Journal of Psychiatry, 159, 276-283. https://doi.org/10.1176/ appi.ajp.159.2.276
Smits, L. M., Feenstra, D. J., Bales, D. L., de Vos, J., Lucas, Z., Verheul, R., y Luyten, P. (2017). Subtypes of borderline personality disorder patients: A cluster-analytic approach. Borderline Personality Disorder and Emotion Dysregulation, 4(16). https://doi.org/ 10.1186/s40479-017-0066-4

Strine, T. W., Balluz, L. S., Chapman, D. P., y Mokdad, A. H. (2008). The associations between life satisfaction and healthrelated quality of life, chronic illness, and health behaviors among U.S. community-dwelling adults. Journal of Community Health, 33, 40-50. https://doi.org/10.1007/s10900-007-9066-4

Üstün, T.B., Chatterji, S., Kostanjsek, N., Rehm, J., Kennedy, C., Epping-Jordan, J., ...von Korff, M. (2010). Developing the World Health Organization Disability Assessment Schedule 2.0. Bulletin of the World Health Organization, 88, 815-823. https://doi.org/10.2471/BLT.09.067231

Van Asselt, A. D. I., Dirksen, C. D., Arntz, A., Giesen-Bloo, J. H., y Severens, J. L. (2009). The EQ-5D: A useful quality of life measure in borderline personality disorder? European Psychiatry, 24, 79-85. https://doi.org/10.1016/j.eurpsy.2008.11.001

Vázquez-Barquero, J. L., Vázquez Bourgón, E., Herrera Castanedo, S., Saiz, J., Uriarte, M., Morales, F.,.., y Grupo Cantabria en discapacidades. (2000). Versión en lengua española de un nuevo cuestionario de evaluación de discapacidades de la OMS (WHO-DAS-II): Fase inicial de desarrollo y estudio piloto. Actas Españolas de Psiquiatría, 28, 77-87.

Vilagut, G., Ferrer, M., Rajmil, L., Rebollo, P., Gaietá, P.M., Quintana, J.M., ...Alonso, J. (2005). El cuestionario de salud SF-36 español: Una década de experiencia y nuevos desarrollos. Gaceta Sanitaria, 19, 135-150. https://doi. org/10.1590/S0213-91112005000200007

Ware, J. E., Snow, K. K., Kosinski, M., y Gandek, B. (1993). Health Survey: Manual and interpretation guide. Boston: New England Medical Center.

World Health Organization (WHO). (2001). World Health Organization Disability Assessment Schedule II (WHODAS II) http://www.who.int/icidh/whodas/index.html. 